\title{
ATOMIC ORDERING AND MARTENSITIC TRANSITION IN A Cu-Zn-AI SHAPE MEMORY ALLOY
}

\author{
J.L. MACQUERON, M. MORIN, G. GUÉNIN, A. PLANES*, J. ELGUETA*and T. CASTAN* \\ GEMPPM and LTSU, INSA, 20 A. Einstein, F-69621 Villeurbanne, France \\ *Departament ECM, Universitat de Barcelona, Diagonal 647, SP-08028 Barcelona, Catalonia, Spain
}

\begin{abstract}
We present results from both, calorimetric and dilatometric studies of the isothermal ordering process taking place in a $\mathrm{Cu}-\mathrm{Zn}-\mathrm{Al}$ shape memory alloy after quenches from $\mathrm{T}_{\mathrm{q}}$ temperatures ranging from $350 \mathrm{~K}$ to $1200 \mathrm{~K}$. The dissipated energy and the length variations of the system are obtained during the process. The change of these quantities in the whole process have been compared with the difference $\Delta \mathrm{M}_{s}$ between $\mathrm{M}_{\mathrm{s}}$, measured after the relaxation and $M_{s}$ measured just after the quench. We obtain that these three quantities present, as a function of $T_{q}$, the same qualitative behaviour. These changes are then associated with changes of the $\mathrm{L} 2{ }_{1}$ ordering after the quench in the system.

The relaxational process does not follow a single exponential decay. Instead, a continuous slowing down is observed. A relaxation time $\tau$ has been defined to characterize the relaxation rate. We show that $\tau$ depends on both the annealing and the quenching $\left(\mathrm{T}_{\mathrm{q}}<800 \mathrm{~K}\right)$ temperatures through an Arrhenius law.
\end{abstract}

\section{1.- Introduction.}

Within a certain range of composition the $\mathrm{Cu}-\mathrm{Zn}-\mathrm{Al}$ alloy system shows a bcc ( $\beta$-phase) structure stable at high temperatures. These alloys exhibit a $\mathrm{B} 2$ superlattice bellow a temperature $\mathrm{T}_{\mathrm{B} 2}$, order to a $L_{2}$, superlattice at $T_{L 21}<T_{B 2}$, and at a lower temperature $M_{s}$, they undergo a thermoelastic martensitic transition [1]. The thermodynamic properties of such transition are very sensitive to the ordering state of the system [2]. For instance, the $\mathrm{M}_{\mathbf{s}}$ temperature might change as much as $50 \mathrm{~K}$ when the degree of atomic order present in the crystal is reduced [3]. The amount of atomic order can be established by annealing the system at a given temperature $\mathrm{T}_{q}$ and then retained (or partially retained) by quenching to a lower temperature. After the quench, at $T_{a}$, the system may evolve towards the equilibrium state corresponding to the new coupling conditions with the surroundings. The study of this relaxational process is of great interest in order to better understand the characteristics of the thermoelastic martensitic transformation.

In $\mathrm{Cu}-\mathrm{Zn}-\mathrm{Al}$ it is known that the $\mathrm{B} 2$-ordering occurs very rapidly and cannot be prevented during the quench from $T_{q}$ so that, in almost all cases, it is already over just after the quench [4]. Thus, the ordering process taking place after the quench, corresponds to the reconstruction of the $\mathrm{L} 2_{1}$ superlattice. This process involves exchanges of atoms among the different lattice sites. These exchanges are assumed to occur via the vacancy mechanism. Since vacancies can be eliminated during the relaxation and their mobility affected as ordering degree changes, the process appears as very complex.

In this work we deal with the study at different $T_{a}$ temperatures of such isothermal relaxational 
process in a Cu- $\mathrm{Zn}-\mathrm{Al}$ alloy after quenches from different $\mathrm{T}_{q}$ temperatures. The ordering process has been followed by measuring the heat dissipated during the process and the evolution of the geometrical dimensions of the sample which are related to changes of the lattice parameter of the crystal. The changes of these magnitudes are compared to the corresponding shift of the $M_{s}$ temperature. From these measurements, the interest is to analyse the role of different parameters as $T_{q}, T_{a}$ and quenching rate on the specific features of the relaxation process and its relevance in relation to the martensitic transformation.

\section{2.- Experimental details.}

\section{1.- Experimental device and samples.}

The energy released during the isothermal relaxation has been measured with a high sensitivity (400 $\mathrm{mV} / \mathrm{W}$ ), fast response, differential conduction calorimeter. At each measurement temperature $\mathrm{T}_{\mathrm{a}}$, the calorimeter was carefully thermalized so that the drift of the baseline was less than $1 \mu \mathrm{W}$ per day. We have followed the evolution of the sample dimensions using an isothermal dilatometer capable to detect length variations smaller than $0.05 \mu \mathrm{m}$. The maximum drift observed (after several weeks with the sample under nominal conditions) was of $0.1 \mu \mathrm{m}$.

The transition temperature, $M_{s}$, has been obtained either by detecting the acoustic emission generated during the martensitic transformation or using a Differential Scanning Calorimeter which, in addition, enables the obtention of the latent heat of the martensitic transformation.

$\mathrm{Cu}-\mathrm{Zn}-\mathrm{Al}$ single crystals (grown by the Bridgman method) and polycrystals elaborated with high purity elements (99.99\%) have been used. We note, however, that results obtained with single and polycrystals are completely equivalent. The nominal composition is $\mathrm{Cu} ; 14$ at\%; 17 at\% $\mathrm{Al}$. The $\mathrm{M}_{\mathrm{s}}$ temperature is $245 \mathrm{~K}$ and the order-disorder transitions temperatures are $\mathrm{T}_{\mathrm{B} 2}=860 \mathrm{~K}$ and $\mathrm{T}_{\mathrm{L} 21}=$ $640 \mathrm{~K}$ [5]. Disk shaped specimens of $13.5 \mathrm{~mm}$ diameter and $1.5 \mathrm{~mm}$ of thickness were used for calorimetric experiments and wires of $40 \mathrm{~mm}$ length and $3 \mathrm{~mm}$ diameter for dilatometric experiments. $M_{s}$ was measured on the same samples used for calorimetric experiments. Additionally, a number of measurements were carried out on thinner samples $(0.1 \mathrm{~mm})$ in order to analyse the influence of quenching rate.

\section{2.-Experimental procedure.}

In order to eliminate any possible influence of previous heat treatments, the sample was, in all experiments, first maintained at $1073 \mathrm{~K}$ for $360 \mathrm{~s}$ and then immersed into a bath at $T_{q}\left(T_{a}<T_{q} \leq\right.$ $1073 \mathrm{~K}$ ) during a time checked to be long enough for the equilibrium degree of atomic order and vacancy concentration at $T_{\mathrm{q}}$ to be reached. The sample was then quenched into an ice-water mixture $(\mathrm{T}=273 \mathrm{~K}$ ) and placed, as quickly as possible, in the microcalorimeter (or in the dilatometer) at the annealing temperature $T_{a}$. The total elapsed time between the quench and the first representative acquisition was typically of $120 \mathrm{~s}$.

\section{3.-Results and discussion.}

In fig.1, for different values of $T_{Q}$, we simultaneously show the total energy $Q=-\int$ Wdt released during the ordering process ( $W$ is the power dissipated at each instant $t$ ), the total relative length change $\Delta L / L$ of the sample, and the difference $\Delta M_{s}$ between $M_{s}(t \rightarrow \infty)$ measured after aging and $M_{s}(t=0)$ measured just after the quench. Measurements have been performed at different $T_{s}$ temperatures $(312 \mathrm{~K}, 302 \mathrm{~K}$ and $281.5 \mathrm{~K})$. It is worth noting that within experimental uncertainties, no differences are revealed between measurements performed at these different $T_{\mathrm{a}}$ temperatures. We observe that these three quantities $\mathrm{Q}, \Delta \mathrm{L} / \mathrm{L}(\Delta \mathrm{L}=\mathrm{L}(\mathrm{t} \rightarrow \infty)-\mathrm{L}(\mathrm{t}=0)<0)$ and $\Delta \mathrm{M}_{\mathrm{s}}$ follow, as a function of $T_{q}$, the same qualitative behaviour. The curves present a pronounced maximum located at the same position $\left(T_{q}-500 \mathrm{~K}\right)$ and low values of the magnitudes for $T_{q}$ 's around $800 \mathrm{~K}$. Actually the three quantities $\Delta M_{s}, Q$ and $-\Delta L / L$ are proportional between them. Notice that an equivalent linear relationship was already reported by Rapacioli \& Ahlers [6] between $\Delta \mathrm{M}_{\mathbf{s}}$ and the relative 
resistivity change of the sample. It is interesting to note that this proportionality between $\mathrm{Q}$ and $\Delta \mathrm{L}$ also holds during the relaxational process at a given $T_{a}$ temperature after a quench from $T_{q}$. In fig.2 we have plotted for $T_{q}=500 \mathrm{~K}$ and $T_{a}=302 \mathrm{~K}, \mathrm{Q}(\mathrm{t}) / \mathrm{Q}$ as a function of $\Delta \mathrm{L}(\mathrm{t}) / \Delta \mathrm{L}$. Here $\mathrm{Q}(\mathrm{t})$ and $\Delta L(t)$ are respectively the dissipated energy and the length change up to a time t. Therefore we conclude that $\mathrm{Q}$ and $\Delta \mathrm{L}$ provide equivalent information relative to the same phenomenon: the ordering process. Nevertheless, from experimental considerations, both kind of measurements complements one to the other in the following sense. During the early stages of the relaxation, the large signal/noise ratio of the calorimetric register permits an excellent definition of the process. This is an important fact because it enables a precise determination of a characteristic time for the process. Oppositely, at very long times the calorimetric signal becomes very small and therefore masked by the noise. As a consequence of this inconvenience, the process cannot be followed. Additionally this poor (or even inexistent) definition of the long time signal renders difficult the evaluation of the total dissipated energy $Q$. It is in this case when the dilatometric technique appears as very suitable to follow the evolution of the system. The total relative change $\Delta \mathrm{L} / \mathrm{L}$ is thus obtained with a good accuracy.

In fig. 3 we show the measured values of $M_{s}(t=0)$ and $M_{s}(t \rightarrow \infty)$ as a function of $T_{q}$. It is interesting to note that the $M_{s}$, measured after aging at $T_{a}$ still depends on $T_{q}$. Since $M_{s}$ depends on the thermodynamic state of the system, this result indicates that the "final state" after the relaxation is different for different quenching $T_{q}$ temperatures and hence that the dissipated energy or the length change are measured in each case in relation to different reference states ("final states"), which in turn depend on $\mathrm{T}_{\mathrm{q}}$.

In order to study the eventual influence of the quenching rate on our experimental results, measurements of $\mathrm{M}_{s}$ just after the quench from $\mathrm{T}_{\mathrm{q}}$ have been performed in very thin samples (see section 2.1 for details). Results are also plotted in fig.3. They show that the effect of a finite quenching rate become relevant for $T_{q}$ temperatures greater than $500 \mathrm{~K}$. This limit temperature is close to the temperature at which $\mathrm{Q}, \Delta \mathrm{L} / \mathrm{L}$ and $\Delta \mathrm{M}_{8}$ display the maximum (see fig. 1 ). The observed decrease of these quantities for higher quenching temperatures is then interpreted as a proof of the existence of a significant ordering taking place already during the quench (time scale for ordering becomes comparable to the typical time scale involved in the quench). Such a partial ordering may be easily understood in terms of a large enough vacancy concentration in this range of $T_{q}$ temperatures. We have verified this explanation by means of a Monte Carlo simulation study of the ordering process during the quench. In these simulations the system was described in terms of an Ising like model and ordering took place via a vacancy mechanism. The vacancy concentration was taken dependent on the $\mathrm{T}_{q}$ temperature.

The analysis of the dissipated thermal power $W$ versus time $t$, unambiguously shows that the relaxation process does not follow a single exponential decay but rather a continuous slowing down is observed. This extends the process to periods of time which are, as indicated previously, of the order of a few days. We have then defined a characteristic time $\tau$ for the relaxation process as the time at which the product t.W shows its most pronounced maximum [7]. In our case, all recorded curves t.W versus $t$ exhibit a well defined maximum. This provides a good reason to justify the procedure used. From an experimental point of view this method of defining $\tau$ is very useful because it is barely affected by the poor knowledge of the dissipated power during the early stages of the process, neither by the long tails displayed by the calorimetric curves. The method is also worthy for making evident the simultaneous contribution of various relaxation times. The ordering process is believed to be assisted by a vacancy mechanism. Thus we assume that $\tau^{-1}$ is proportional to the initial equilibrium vacancy concentration at $T_{q}$ and to the vacancy mobility at $T_{0}$. Consequently, we consider that $\tau$ depends on $T_{8}$ and $T_{q}$ through an Arrhenius law:

$$
\tau=A \exp \left(\frac{E_{f}}{k T_{q}}\right) \exp \left(\frac{E_{m}}{k T_{a}}\right)
$$

$E_{1}$ and $E_{a}$ are respectively the effective vacancy formation and migration energies. Fig. 4 shows that 


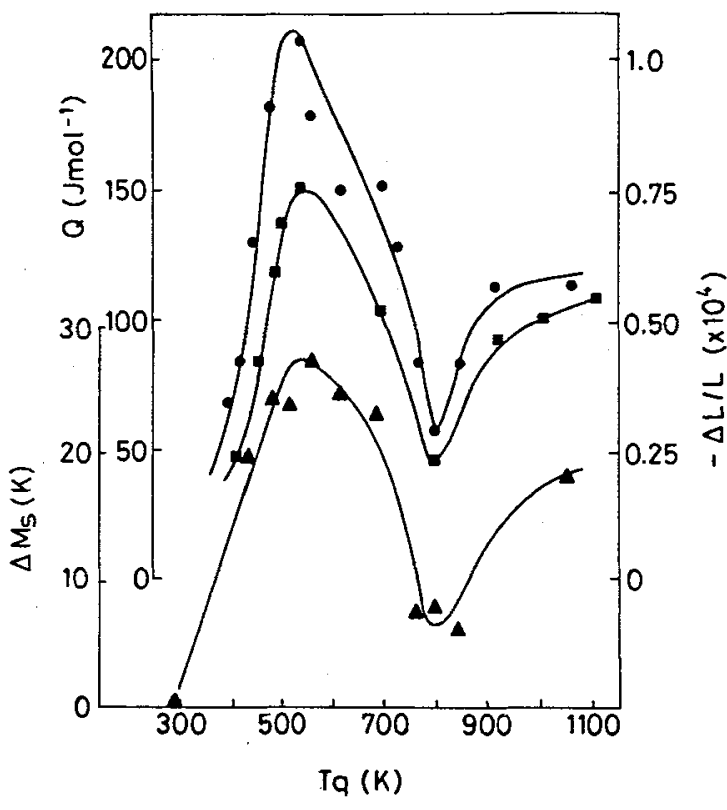

Fig.1 Dissipated energy ( $\bullet$, relative change of length (E) and shift of the martensitic transition temperature $(\Delta)$ as a function of $\mathrm{T}_{\mathrm{q}}$. Lines are guides to the eyes.

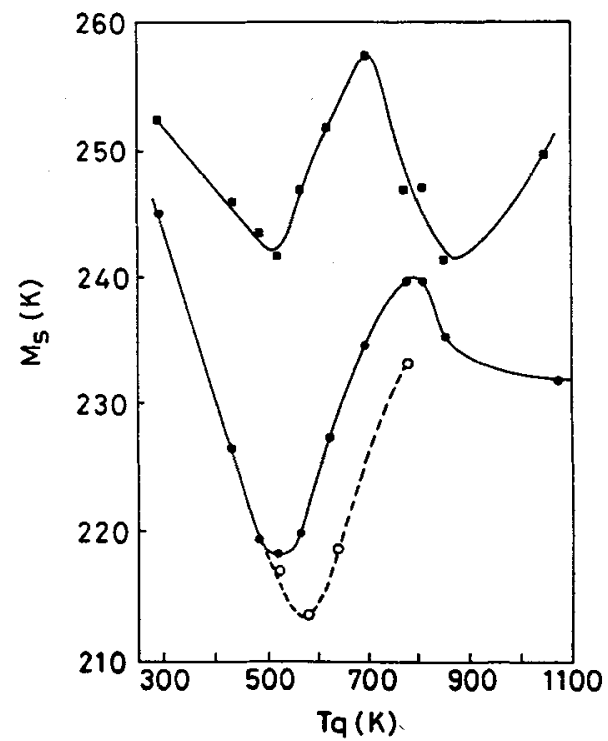

Fig.3 Martensitic transition temperature $M_{s}$ obtained immediately after the quench ( ), and after the relaxation process ( $\bullet$ ). (o) are the values obtained just after the quench using a very thin sample. Lines are guides for the eyes.

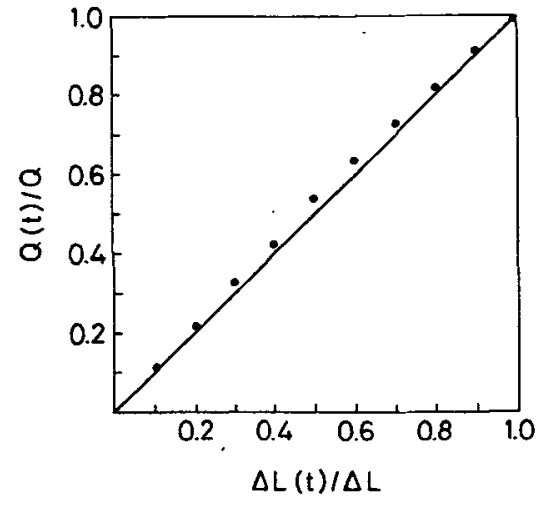

Fig.2 Normalized dissipated energy versus the normalized length change at different times $t$ of the relaxation.

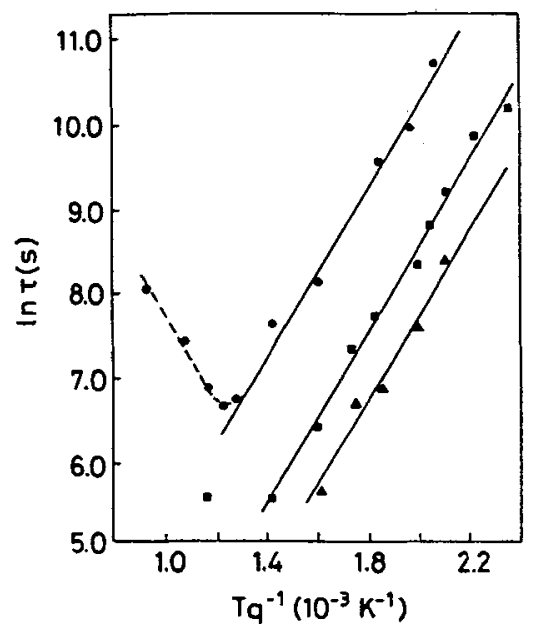

Fig. $4 \ln \tau$ versus $T_{q}^{-1}$ for $T_{a}=312 K(\Delta), 302 K$ $(\varpi)$ and $281.5 \mathrm{~K}(\bullet)$. Continuous lines are linear fits to the experimental points. 
$\tau$ indeed obeys such law, except for $T_{q} \geq 800 \mathrm{~K}$. Linear fits $\left(T_{q}<800 \mathrm{~K}\right)$ give $E_{m}=0.76 \pm 0.03 \mathrm{eV}$ and $\mathrm{E}_{8}=0.43 \pm 0.03 \mathrm{eV}$.

Looking at fig. 4 it becomes clear that irrespective of $T_{a}, \tau$ presents a minimum for $T_{q}$ close to 800 $\mathrm{K}$. This result could be interpreted as a proof of a partial elimination of effective vacancies during quenches from $T_{Q}>800 \mathrm{~K}$. Since $T_{B 2}$ is close to such temperature, we suggest that this elimination of effective vacancies may be due to its trapping at antiphase boundaries of $\mathrm{B} 2$ ordered domains which form during the quench. Moreover, it should be pointed out that if this vacancy elimination becomes sufficiently relevant, a much less important reordering should occur during the quench. This is consistent with the increase of the $\mathrm{Q}, \Delta \mathrm{L} / \mathrm{L}$ and $\Delta \mathrm{M}_{s}$ for quenches from $\mathrm{T}_{\mathrm{q}}>800 \mathrm{~K}$.

Therefore, we might, from our results, finally conclude, that giving a quenching procedure, the configurational ordering state at $T_{q}$ is effectively frozen by the quench only when $T_{q}$ is lower than the temperature at which $\mathrm{Q}$ (or $\Delta \mathrm{L} / \mathrm{L}$ or $\Delta \mathrm{M}_{\mathrm{s}}$ ) exhibit a maximum, while the temperature range enabling the freezing of the vacancy concentration extends up to $T_{\mathrm{B} 2}$.

\section{Acknowledgements.}

AP acknowledges the Dirección General de Investigación Cientifica y Técnica (Ministerio de Educación, Spain) and the Direction de la Coopération Scientifique (Ministère des Affaires Etrangères, France) for financial support during his stay at Villeurbanne. Partial fantail support from Comisión Interministerial de Ciencia y Tecnología (Spain) under project MAT89-0748 is also acknowledged.

\section{References.}

1. M.Ahlers, Prog.Mater.Sci. 30 (1986) 135

2. H.C.Tong, C.M.Wayman, Acta Metall. 23 (1975) 209

3. A.Planes, R.Romero, M.Ahlers, Acta Metall. 38 (1990) 757

4. T.Suzuki, Y.Fujii, A.Nagasawa, Materials Science Forum, 56-58 (1990) 481

5. A.Planes, Ll.Mañosa, E.Vives, J.Rodríguez-Carvajal, M.Morin, G.Guénin, J.Phys: Condens. Matter, to be published.

6. R.Rapacioli, M.Ahlers, Acta Metall. 27 (1979) 777

7. J.Elgueta, J.L.Macqueron, A.Planes, J.Phys.:Condens. Matter, to be published. 